\title{
IONOSPHERIC FOCUSSING
}

\author{
by G. J. Daniell \\ (Cavendish Laboratory, Cambridge, England)
}

R ́́sumட́. - Une antenne placée dans l'ionosphère ne peut recevoir de rayonnement extraterrestre qu'àl'intérieur d'un cône déterminé. On résume brièvement les calculs théoriques utilisant des modèles ionosphériques variés qui donnent les dimensions et la forme du cône et l'intensité du signal reçu dans le cas d'un ciel uniformément brillant et d'une source ponctuelle unique. On discute l'approximation de l'optique géométrique, ainsi que l'effet de la courbure de la Terre et du champ magnétique terrestre.

ABSTRACT. - A radio antenna within the ionosphere can receive extraterrestrial radiation incident only within a limited cone of directions. A brief review is presented of the theoretical calculations, using various models, of the size and shape of the cone, and the magnitude of the received signal, both from a uniformly bright sky and from a single point source. The approximations of ray theory are discussed and the effects of the curvature of the earth and the inclusion of the terrestial magnetic field are mentioned.

Г'езюме. - Антенна помещенная в ионосферу может получать внеземное излучение лишь внутри отределенного конуса. Дана краткая сводка теоретических вычислений, использующих разли'ные модели ионосферы, даюшие размеры и форму конуса и интенсивность сигнала, полученного в случае равномерно блестящего неба и единственного точечного источника. Обсуждено приближение геометрической оптики, как и эффект кривизны земли и земпого магнитного поля.

\section{IntRoduction.}

In recent years considerable interest has been shown in the possibilities of extending radioastronomical observations to lower frequencies by using a satellite or rocket borne receiver, above the level of the maximum electron density in the ionosphere. In this way measurements can be extended to frequencies of the order of $1-5 \mathrm{MHz}$ which do not normally penetrate to the surface of the earth. A useful value of the spectral index of the radiation at these frequencies can be found from measurements of the average brightness of the whole sky. If some angular resolution can be obtained, it is then possible to study individual features of the radio emission [1]. Several methods have been suggested for using the refracting properties of the ionosphere to obtain directivity (for example, the use of the $\mathrm{Z}$ mode proposed by ELLIS [2]). The simplest method uses the property that, for radio waves, the refractive index of the ionosphere is neariy always less than unity, so that rays incident only within a limited cone can reach a receiver. The object of this paper is to give a brief review of this method.

The radioastronomer needs to know the overall polar diagram of his aerial system, that is the com- bined effect of the ionosphere and the antenna. It can be assumed that the output of the receiver can be related to the electric or magnetic fields within the ionosphere, and this paper describes the calculations relating these to the intensity of the incoming radiation, for various ionospheric models.

If the cone angle is small, the sky radiation may be taken as uniform over the cone and the fields produced under such conditions can be calculated on thermodynamic grounds, as well as by integration of the fields of a single ray over all incident ray directions. In Sections 2 and 3 the ionosphere is assumed to be isotropic and horizontally stratified. Section 2 reviews the ray theory treatment and Section 3 the "full wave" analysis. Section 4 discusses the effects of the receiver bandwidth on observations using the focussing effect. The complications introduced by the inclusion of the curvature of the earth are reviewed in Section 5, and some of the effects introduced by the terrestrial magnetic field are mentioned in Section 6.

\section{Horizontalliy \\ STRATIFIED ISOTROPIC IONOSPHERE.}

If we assume for the moment that the ionosphere is a homogeneous, isotropic, horizontally stratified medium whose refractive index decreases 
monotonically from a value $n_{0}$ at the receiver to unity at great distances, then a ray incident on the ionosphere at an inclination $\theta$ to the vertical is refracted so that it has an inclination $\psi$ at the satellite given by Snell's Law

$$
n_{0} \sin \psi=\sin \theta \text {. }
$$

For a given $\theta$ two ray paths to the receiver are possible, one direct and the other reflected below the level of the satellite. As $\theta$ increases the two rays become closer together until for a value of $\theta, \theta_{0}$ given by $\sin \theta_{0}=n_{0}$, the rays are both horizontal at the receiver. Rays incident at angles $>\theta_{0}$ cannot reach the receiver, and hence a natural mechanism for producing directivity exists ; moreover it will be shown below that the rays incident near the edge of the cone $\theta=\theta_{0}$ produce a large signal in the ionosphere, the so called " edge focussing effect". The fields are easily found by noting that, for the W. K. B. solutions of the equations for wave propagation in the ioncsphere, the vertical component of the Poynting Flux is a constant, if the medium is loss free [3]. In a medium of refractive index $n$ this flux is proportional to $n:\left.\mathbf{E}\right|^{2}$ where $\mathrm{E}$ is the electric field of the wave, so that

$$
n|\mathrm{E}|^{2} \cos \psi=\left|\mathrm{E}_{0}\right|^{2} \cos \theta
$$

$\mathrm{E}_{0}$ being the electric field of the incident wave.

Using (1) the "effective polar diagram" $P(j)$ can be written

$$
\text { (3) } \quad \mathrm{P}(\theta)=\frac{|\mathrm{E}|^{2}}{\left|\mathrm{E}_{0}\right|^{2}}=\frac{\cos \theta}{\left(n_{0}^{2}-\sin ^{2} \theta\right)^{1 / 2}} \text {. }
$$

An important point is that no electron density profile need be specified, a result which is not true if the earth's curvature is included.

According to (3) the electric field is infinite for $\sin \theta=n_{0}$, but this is only a result of the failure of the W. K. B. approximation. The exact full wave treatment is discussed in the next section. For the integrated fields prcduced by a uniformly bright sky the ratio of the values of $|\mathbf{E}|^{2}$ within the ionosphere and in the free space outside the earth is

$$
\begin{aligned}
\int_{0}^{\theta_{0}} \mathrm{P}(\theta) \sin \theta & d \theta \\
& =\int_{0}^{\pi / 2} \frac{\cos \theta \sin \theta}{\left(n_{0}^{2}-\sin ^{2} \theta\right)^{3 / 2}} \frac{d \theta}{d \psi} d \psi=n_{0}
\end{aligned}
$$

which agrees with the known result that the thermal equilibrium radiation in a medium is proportional to the refractive index.

\section{ISOTROPIC IONOSPHERE, Full Wave tren'tment.}

Budden [4] has shown that if the electron density can be assumed to vary linearly with height in the region of the satellite, then the electromagnetic fields are given by Airy functions or their derivatives. For example the electric field perpendicular to the plane of incidence is proportional to

$$
\mathrm{A}_{\mathrm{I}}\left\{\left(n_{0}^{2}-\mathrm{in}^{2} 0\right)(k / \alpha)^{: / 3}\right\},
$$

where $k$ is the free space wave number and $\alpha$ is a measure of the electron density gradient defined by $n^{2}=n_{0}^{2}+\alpha z$. This shows that although the signal is large when $\theta \sim \theta_{0}$, it remains finite, and also that a small signal can reach the receiver for angles $>\theta_{0}$. The full wave analysis also shows that interference effects occur between the direct rav and the reflected ray so that the signal as a function of $\theta$ shows oscillations or fringes, the envelope being given approximately by (3).

\section{THE EFFECT OF THE BANDWIDTH OF THE RECEIVTR}

The refractive index of the ionosphere is given by $n^{2}=1-f_{\mathrm{N}}^{2} / f^{2}$ where $f_{\mathrm{N}}$ is the plasma frequency and this may be a rapidly varying function of the observing frequency $f$. All the previous results apply only at a single frequency, whereas the output of the receiver depends on the sum of the fields at frequencies within the baudpass of the receiver. For the mcdel of Section 2 the modified polar diagram is

$$
\int \mathrm{P}(0, f) g(f) d f
$$

where $g(f)$ is the shape of the receiver bandpass. If we take $g(f)=1$ for

$$
\left|t-t_{0}\right|<\Delta t_{0}
$$

and $g(f)=0$, otherwise, this is

$$
\int_{t_{0}-\Delta t_{0}}^{t_{0}+\Delta t_{0}} \mathrm{P}(0, f) d f
$$

It is easily shown that the infinity in the received signal is removed, the maximum signal still occurring for $\theta \simeq \theta_{0}$ but having value $\simeq\left(\frac{1}{2} \Delta\right)^{-1 / 2}$ typically $\sim 10$.

The bandwidth effect will also tend to blur the fringes described in 3 . If the path difference 
between the direct and reflected rays varies by a wavelength over the bandwidth of the receiver the fringe system will vanish. This can be shown to occur if $\frac{\mathrm{K} \Delta}{\alpha} \sim 1$.

\section{FfFects of the earth's CURVATURE.}

As would be expected the general features of the problem are not changed by the introduction of this complication. The direct and reflected rays now no longer reach the satellite at supplementary angles, the extreme ray at the cone edge is not horizontal at the satellite, and the polar diagram now depends on the electron density profile.

Some calculations of the fields are given by HASELGROVE et al. [5] for an experimentally observed profile.

The full wave analysis has been discussed by BUDDEN [4] and further mathematical techniques and numerical calculations for an exponential profile are being studied.

The integrated fields from a uniformly bright sky must, by thermodynamic reasoning, be the same as for a flat earth.

\section{FfFects of the earth's Magnetic hleid.}

The terrestrial magnetic field renders the ionosphere anisotropic and greatly complicates the problem. Firstly the ray entering the ionosphere is split into ordinary and extraordinary rays which are reflected at different heights. Secondly, the system no longer has rotational symmetry about the vertical, and so the incident ray direction must be specified by two parameters $(\theta, \varphi)$, $\%$ being the azimuth. It can be shown however that the fields produced by incident ravs symmetric with respect to the magnetic meridian plane are the same, as also is the sum of the fields of the direct and reflec- ted rays arising from rays symmetric about a plane perpendicular to the magnetic meridian. It is easily shown moreover that the inclination of the extreme ordinary ray incident perpendicular to the magnetic meridian is the same as in an isotropic medium and that for incidence in the magnetic meridian the inclination is greater.

In an isotropic medium the direction of the electric and magnetic vectors bears a simple relation to the ray direction. If a magnetic field is present however it is necessary to calculate three components of the field vectors, as a function of incident ray direction. Papagiannis and $\mathrm{HU}$ GUENIN [6] have calculated the total intensity of the radiation in $\mathrm{W} \mathrm{H}_{z}^{-1}$ Sterad $^{-1}$ on the assumption that the refractive index surfaces can be approximated by ellipsoids of revolution, which enables the general behaviour to be discussed.

Considerably more is known about the integrated fields ; BudDwn and Hugris [7] showed how the field components vary with ionospheric parameters. Considerable deviations from the results for an isotropic medium occur.

\section{Conclusion.}

This paper has reviewed the applications and limitations of ionospheric focussing in radioastronomical observations. The behaviour of the ionosphere in a practical experiment will be much more complex than in any of the models described here, but although complications such as ionospheric irregularities and absorption have been entirely ignored the general features of the behaviour would be unlikely to be greatly changed.

The author is indebted to Prof. F. G. Smrth for helpful discussion of the problem and to the Department of Scientific and Industrial Research for a maintenance grant.

Manuscrit reçu le 20 août 1964.

\section{REFERENCES}

[1] Sмiтн F. G., 1961, Monthly Notices R. A. S., 122. 527.

[2] ElLIS G. R., 1962, Nature, 193, 862.

[3] Budden K. G., Radio waves in the ionosphere Cambridge University Press. Ch. 9.

[4] Budden K. G., 1961, Proc. Roy. Soc., 263, 552.
[5] Haselgrove C. B., Haselgrove J. and Jennison R. C., 1961, Proc. Roy. Soc., 261, 423.

[6] Papagiannis M. D. and Huguenin G. R., 1964, J. G. R., 69, 1307.

[7] Buddex K. G. and Hugrid, J. 1964, Proc. Roy. Soc., 277365.

\section{Discussion}

R. C. Jenisson. - The information which we have been given makes me more convinced that the technique of earth occultation is much to be preferred to narrow angle focussing. It requires a satellite in a distant orbit but lends itself to simple electronic techniques (e. g. frequency switching) for the removal of the background. I shall be very interested to hear if Prof. HADDOCK or Dr. STEINB ERG have considered using this technique in future vehicles. 